\title{
Muséologies
}

Les cahiers d'études supérieures

\section{La collection muséale et la variabilité de l'oeuvre : échantillon de pratiques et rôle de l'artiste}

\section{Véronique Rodriguez}

Volume 5, numéro 1, automne 2010

URI : https://id.erudit.org/iderudit/1033524ar

DOI : https://doi.org/10.7202/1033524ar

Aller au sommaire du numéro

\section{Éditeur(s)}

Association Québécoise de Promotion des Recherches Étudiantes en Muséologie (AQPREM)

ISSN

1718-5181 (imprimé)

1929-7815 (numérique)

Découvrir la revue

Citer cet article

Rodriguez, V. (2010). La collection muséale et la variabilité de l'oeuvre : échantillon de pratiques et rôle de l'artiste. Muséologies, 5(1), 108-134. https://doi.org/10.7202/1033524ar
Résumé de l'article

Pour de nombreux artistes, les années 1960 marquent un tournant dans leur pratique au cours duquel ils choisissent de délaisser les médias de création traditionnels : gravure, peinture ou sculpture pour se tourner vers de nouveaux médiums au caractère variable : la performance, l'installation ou la vidéo. L'entrée de ces oeuvres dans les collections muséales entraîne son lot de changements. En analysant et comparant les pratiques au sein de deux institutions nationales, l'une au Québec (MBAQ) et l'autre en Suisse (Mamco), Rodriguez démontre que l'authenticité de l'oeuvre ne repose plus uniquement sur sa matérialité, mais bien sur l'intention de l'artiste, devenue une valeur culte. 
Véronique Rodriguez

\section{La collection muséale et la variabilité de l'œuvre : échantillon de pratiques et rôle de l'artiste}

Pour de nombreux artistes, les années 1960 marquent un tournant dans leur pratique au cours duquel ils choisissent de délaisser les médias de création traditionnels : gravure, peinture ou sculpture pour se tourner vers de nouveaux médiums au caractère variable: la performance, l'installation ou la vidéo. L'entrée de ces œuvres dans les collections muséales entraîne son lot de changements. En analysant et comparant les pratiques au sein de deux institutions nationales, l'une au Québec (MBAQ) et l'autre en Suisse (Mamco), Rodriguez démontre que l'authenticité de l'œuvre ne repose plus uniquement sur sa matérialité, mais bien sur l'intention de l'artiste, devenue une valeur culte.

Véronique Rodriguez est professeure d'histoire de l'art au Collège Ahuntsic à Montréal (Québec) depuis 1999 et professeure associée à l'UQAM. Elle a obtenu un doctorat en sociologie de l'Université de Montréal en 2001. Ses recherches portent principalement sur la sculpture et l'installation, les pratiques d'atelier et d'exposition de l'art contemporain.VERONIQUE.RODRIGUEZ@VIDEOTRON.CA 


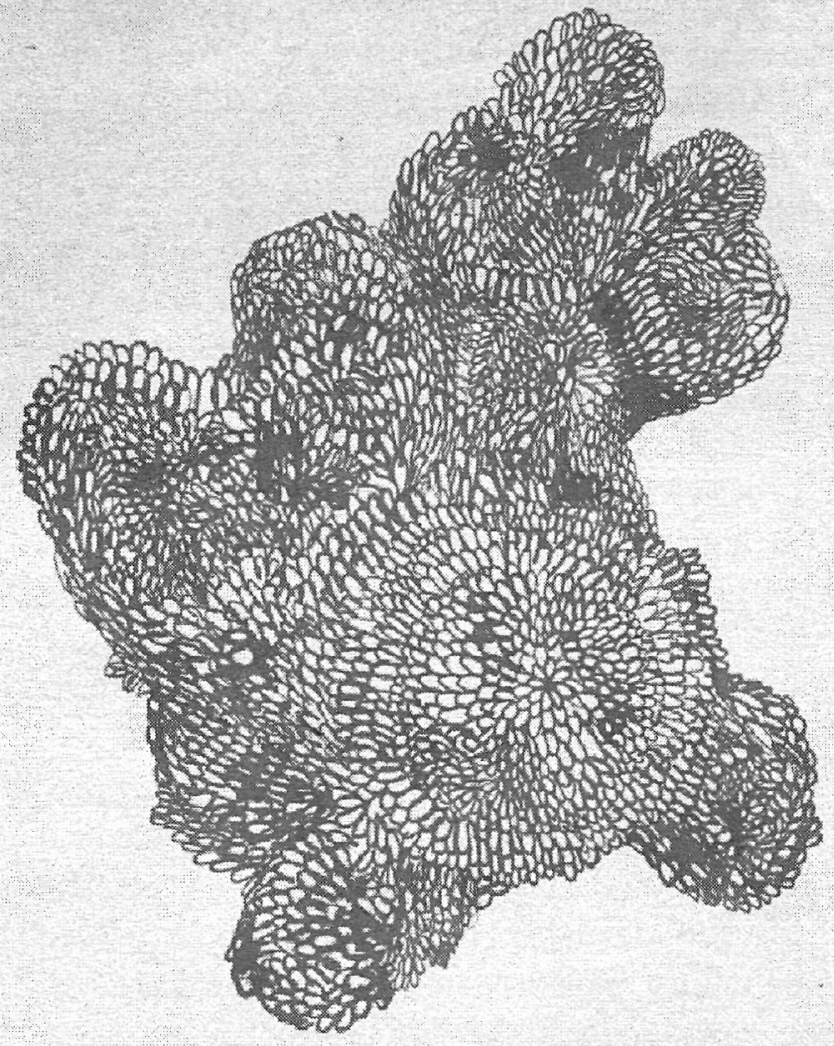


Nous avons entrepris cette réflexion dans notre thèse de doctorat, L'exacerbation de la valeur d'exposition et la dévalorisation du métier de l'artiste, leurs incidences sur les transformations de l'atelier. (Université de Montréal, 2001,461 p.). Nous poursuivons nos recherches au sein du projet dirigé par Francine Couture (Département d'histoire de l'art, UQAM) intitulé Réexposition, réactualisation et pérennité des œuvres contemporaines, 2006-2011, subventionné par le CRSH.
La remise en question des pratiques artistiques depuis les années 1960 a conduit beaucoup de créateurs à investir d'autres formes artistiques telles que la performance, l'installation, la vidéo, etc. Fréquemment associée à son contexte d'exposition, l'œuvre nécessite des gestes de création à chaque fois qu'elle est montrée. Ce faisant, l'artiste se trouve à déplacer une partie de la production de l'œuvre de l'atelier à la salle d'exposition. La production n'est plus une phase finie quand l'œuvre quitte l'atelier. Elle est devenue inséparable de la diffusion, réactivée périodiquement à chaque exposition où l'artiste s'attribue par le fait même une place non négligeable'.

Ces œuvres, qui sont entrées dans les collections institutionnelles, ont rapidement fait surgir des problèmes d'exposition et de conservation car elles remettent en question des pratiques muséologiques auparavant basées sur des disciplines traditionnelles comme celles de la gravure, de la peinture et de la sculpture. Comment assurer la préservation de l'intégrité de l'œuvre variable d'une exposition à l'autre lorsque la production est réactivée? Et quel est le rôle de l'artiste dans ce processus?

Notre recherche a fait ressortir qu'il n'y a pas de règles muséologiques précises pour répondre à ces questions. Le phénomène étant relativement récent, chaque œuvre est un cas d'espèce dans lequel on prend en compte la culture institutionnelle avec les protocoles mis en place, lorsqu'il y en a, ainsi que le type de collaboration engagé avec l'artiste.

Cependant, en multipliant les études de cas, nous avons $\mathrm{pu}$ dégager des ressemblances et regrouper des pratiques muséographiques. Deux grandes tendances se dessinent. La première réunit des œuvres qui ont varié avant leur acquisition, mais que le processus institutionnel fixe définitivement en instaurant des paramètres de réexpositions astreignants. La seconde réunit des œuvres variables dans leurs occurrences avant et après l'acquisition et dont la production perdure au cours de leur vie institutionnelle, qu'elle soit déléguée ou exécutée par l'artiste. 
Faute de place dans cet article, nous ne présenterons pas d'analyse d'œuvres de la première tendance, bien qu'elle soit la plus courante dans le milieu muséal. En effet, l'œuvre, qui n'est plus variable, se rapproche des usages connus de conservation/restauration des collections muséales. L'œuvre est fixée en collaboration, convoquant plusieurs médiateurs tels l'artiste, le conservateur, le restaurateur, des techniciens ainsi que des spécialistes, selon le cas à l'étude, qui s'entendent sur une présentation qui respecte son intégrité. L'acquisition devient un moment privilégié de la carrière de l'œuvre, moment où elle adopte une forme qui marque une rupture avec son histoire.

Nous présenterons ici la seconde tendance, celle des œuvres qui conservent leur variabilité, à partir d'études de cas exemplaires. Dans cet article, nous nous intéresserons exclusivement à des œuvres collectionnées, appartenant à des institutions publiques. Les œuvres que nous analyserons appartiennent à deux musées, un québécois et un suisse : le Musée national des beaux-arts du Québec (MNBAQ) à Québec et le Musée d'art moderne et contemporain (Mamco) à Genève. Dans un premier temps, nous présenterons trois œuvres, toutes conservées au MNBAQ, qui témoignent de la délégation de la production aux professionnels du musée: many things were left unsaid (2003-2004) de Karilee Fuglem, Capteurs d'essence (1994) d'Annie Thibault et Darboral (2000-2005) de Massimo Guerrera. Avec L'Atelier depuis 19380 (1994) de Sarkis au Mamco, nous aborderons le second aspect de la variabilité de l'œuvre avec un cas plus rare, celui où l'artiste en personne poursuit l'œuvre dans son espace, sur place, depuis 1994.

\section{Des œuvres variables : la délégation du faire}

\section{Karilee FUGLEM, many things were left unsaid, 2003-2004, MNBAQ}

La naissance de many things were left unsaid (ill. 1) est indissociable de l'espace d'exposition de Gairloch Gardens des Oakville Galleries, à Oakville en Ontario. 
Illustration 1

Karilee Fuglem, many things were left unsaid, 2003-2004, lettres en vinyle, dimensions variables (selon la citation). Musée national des beauxarts du Québec, 2005.54, don de l'artiste. [Photo: Patrick Altman]

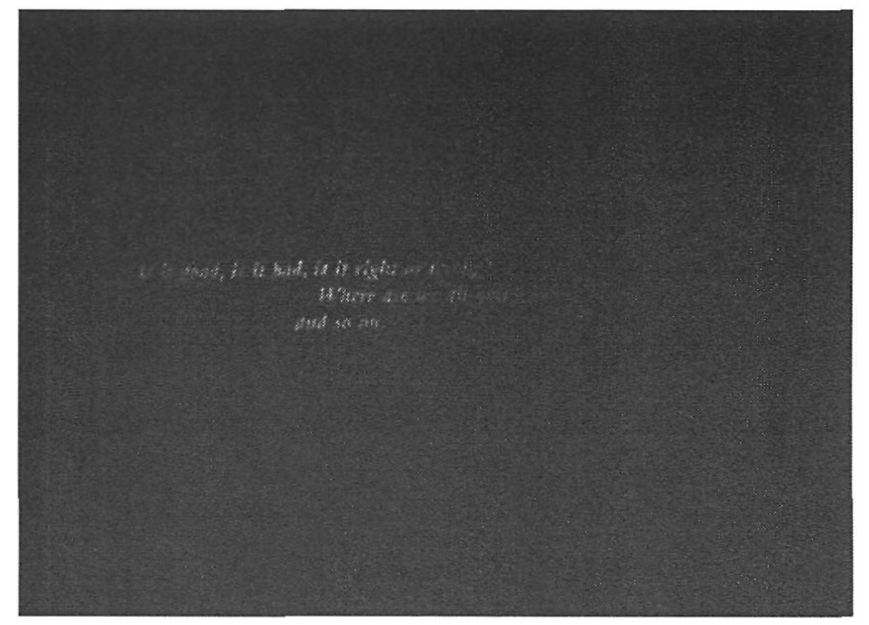

L'architecture de style "cottage anglais » et l'atmosphère de la maison ont rappelé à Karilee Fuglem l'ambiance du roman de Virginia Wolfe, To the Lighthouse. Many things were left unsaid se compose de citations du livre qu'elle a directement collées sur les murs blancs avec des lettres de vinyle blanc. Les reflets de la lumière naturelle sur le plastique, qui change au cours de la journée, attirent l'attention sur ces phrases que le spectateur décèle puis se met à chercher en se déplaçant d'une salle à l'autre. Étant presque toutes placées à la même hauteur mais pas aux mêmes endroits, elles introduisent un aspect ludique à la visite de l'exposition.

C'est dans ce contexte qu'Anne-Marie Ninacs, qui prépare alors l'exposition Avancer dans le brouillard, voit many things were left unsaid et qu'elle propose à l'artiste de l'exposer au MNBAQ. Ce déplacement ne semble pas aller de soi pour Karilee Fuglem qui montre beaucoup d'hésitation. Une correspondance électronique soutenue s'engage alors (disponible dans le dossier de l'œuvre au MNBAO). Au cours de cet échange, on voit progressivement l'artiste circonscrire le concept de l'œuvre, en dehors de sa première exposition, grâce aux discussions avec la conservatrice. Ce retour dans la production débouche sur une réactualisation de l'œuvre pour son nouveau cadre que sera le MNBAQ. 
Non seulement many things were left unsaid participe à l'exposition Avancer dans le brouillard, mais elle est également acquise par le MNBAQ en $2005^{2}$. Les différences entre l'œuvre exposée à Oakville en Ontario, un espace domestique d'inspiration britannique transformé en galerie, et celle à Québec, dans une institution muséale francophone, sont nombreuses. Les citations retenues, qui étaient liées au contexte domestique, changent pour privilégier l'intériorité. Les phrases, qui étaient en anglais, apparaissent dans les deux langues au musée. Choisies par l'artiste dans une édition anglophone du roman, elles sont repérées dans une édition française par la conservatrice afin de trouver leur équivalence et de s'adapter davantage au public de l'institution ${ }^{3}$. Les deux langues se distinguent sur les murs du MNBAQ par la luisance du vinyle, mat ou brillant, mais toujours aussi discrètement sur la cimaise. Les phases ne sont plus éparpillées dans l'espace d'exposition, elles sont réunies sur une longue cimaise, sans pour autant mettre bout à bout les traductions. La police de caractères est identique dans les deux lieux, mais sa taille varie à Québec. Enfin, l'exposition au musée ayant lieu en hiver où il fait nuit à 16 heures, il a fallu y adapter un éclairage artificiel afin que les spectateurs puissent découvrir, au cours de leur déambulation dans l'espace, un effet de brillance sur la cimaise qui manifeste la présence de quelque chose, renforcé par un cartel. L'œuvre si discrète a été très remarquée, comme en témoignent les commentaires de la conservatrice et sa présence dans la revue de presse ${ }^{4}$. Au MNBAQ, many things were left unsaid diffère de sa première apparition, elle est réactualisée et le sera à chaque exposition puisque les lettres de vinyle, une fois posées sur le mur, ne peuvent être réutilisées. Il faut les produire à chaque fois que l'œuvre est montrées.

A la suite de cette deuxième exposition, Karilee Fuglem rédige, à la demande de la conservatrice, un document d'accompagnement qui décrit les contextes d'apparition de l'œuvre à Oakville et à Québec ainsi que ses limites pour des expositions ultérieures ${ }^{6}$. On y trouve une description générale de many things were left unsaid et des notes sur les deux expositions. Y sont ajoutées des consignes qui fixent
2

Une autre œuvre de Karilee Fuglem sera acquise après cette exposition, il s'agit de Untitled (invisible thread) / Sans titre (fil invisible), 2002-2004, fil de nylon, dimensions variables: masse centrale: $100 \times 110 \times 105 \mathrm{~cm}$; avec les fils porteurs: $242 \times 478 \times$ $358 \mathrm{~cm}$. Musée national des beauxarts du Québec, Québec, CP.2005.11. Elle présente des variations similaires à many things were left unsaid avec des réactualisations et des adaptations.

3

Virginia Wolfe: romans et nouvelles (1917-1941). Traduit de l'anglais par MERLE, Magali. Paris: Le livre de poche (Classiques modernes), 1993 4

Entrevue non publiée avec AnneMarie NINACS. 2 mars 2005. Pour la revue de presse, voir BEAUPRE, Marie-Ėve. Avancer dans le brouillard, Musée national des beaux-arts du Québec, Québec, Para-Para, n²0. <http://www.parachute.ca/ para_para/20/parazo_Beaupre. html> (consulté le 12 juillet 2010); BOUCHARD, Julie. "Présent du singulier». Voir (Québec), 4 novembre 2004, p. 21; DELGADO, Jérôme. "Brouillard à voir". La Presse, 12 décembre 2004, Arts et spectacles, p. 12; MANDELSTAM. Avancer dans le brouillard. <http:// quebec.fluctuat.net/blog/ Sentiers+qui+bifurquent.html> (consulté le $n$ juillet 2010). 5 Cette caractéristique de l'œuvre est notée par le restaurateur du MNBAQ, Claude BELLEAU. ("Rapport d'état de conservation". 11 juillet 2005, 1 p. Musée national des beaux-arts du Québec, Québec, dossier 2005-54.)

6

FUGLEM, Karilee. "Notes on Installation of many things were left unsaid ". Septembre 2005, 4 p. Musée national des beaux-arts du Québec, Québec, dossier 2005-54. 
7

Musée national des beaux-arts du Québec, Québec, du 24 avril 2008 au 8 février 2009. Commissaire: Mélanie Boucher. la police de caractères, sa taille, les modèles de vinyle, la hauteur des citations sur les murs, l'installation sur les murs ainsi que le choix des citations extraites du livre. L'artiste prévoit trois versions différentes de l'œuvre, dénommées: "basic", "expanded " et "original», distinguant la première exposition à Oakville, dite originale, des autres, tout en laissant au commissaire la possibilité de ne pas utiliser toutes les citations de chacune des versions.

C'est en collaboration que l'œuvre in situ est réactualisée. L'œuvre est conçue pour être variable et s'adapter à chaque nouveau contexte à partir de ses expositions antérieures posées en référence plutôt que d'exister dans un seul lieu, celui qui l'a vu naître et de disparaître du circuit de diffusion ensuite. Grâce à la collaboration et à la production de son document descriptif, Karilee Fuglem définit le concept de l'œuvre et transmet son savoir-faire. many things were left unsaid peut théoriquement demeurer variable et conserver son intégrité, tout en entrant dans une collection.

Peu après, many things were left unsaid est choisie par la commissaire Mélanie Boucher pour participer au corpus d'Intrus/Intruders en 20087. Dans cette exposition, elle incite les spectateurs à revisiter la collection permanente du MNBAQ en y intégrant des œuvres contemporaines. Quatre citations bilingues liées au rapport de l'homme à l'altérité sont apposées sur les murs de la salle 11, celle de la collection d'art inuit Brousseau - Salle Hydro-Québec. Contrairement aux expositions antérieures, Karilee Fuglem ne s'est pas déplacée pour ce montage. Une fois le choix des phrases approuvé en fonction du thème du lieu, soit l'environnement et les changements climatiques, les consignes du document qu'elle a rédigé ont fourni toutes les indications nécessaires aux techniciens du musée. Cette application montre que le concept de l'œuvre est circonscrit et que l'artiste a véritablement délégué son savoir-faire.

\section{Annie THIBAULT, Capteurs d'essence, 2004, MNBAQ}

L'œuvre les Capteurs d'essence (ill. 2-3) d'Annie Thibault demande à être réactualisée à chaque présentation. Mais 
celle-ci diffère de many things were left unsaid, tout comme sa variabilité dans la collection.

Capteurs d'essence est une œuvre qui fait partie d'un projet plus vaste intitulé La chambre des cultures, exposé pour la première fois en $1996^{\circ}$. Dans le cadre de ce projet, l'artiste travaille avec des micro-organismes vivants qu'elle expose dans des éprouvettes en verre de toutes sortes de formes qu'elle place dans différents contextes. En ce qui concerne les Capteurs d'essence, Annie Thibault n'effectue pas d'inoculation en laboratoire. Une préparation de gélose est placée dans les fioles de formes organiques suspendues par leur queue dans l'espace d'exposition. Les spores et les bactéries de l'air ambiant s'y développent, sèchent et meurent lorsqu'elles ont épuisé la nourriture de l'agar-agar.

Exposée pour la première fois en 1997, chacune des occurrences de la série des Capteurs d'essence s'est adaptée à son contexte. Les variations se font principalement sur le nombre de fioles exposées (de une à sept), sur le mode d'accrochage (en extérieur sur des branches d'arbres ou en intérieur sur des tiges en acier); lorsqu'ils sont en intérieur, les types de support en acier et les crochets changent également, tout comme leur disposition dans l'espace et leur hauteur. Les fioles peuvent être exposées seules, mais le plus souvent elles sont assemblées avec d'autres œuvres du projet. Enfin, Capteurs d'essence faisant intervenir des spores et des bactéries de l'air ambiant, chaque lieu d'exposition produit des cultures différentes dans la gélose. La variation est inscrite au concept même de l'œuvre en travaillant avec des organismes vivants.

Les Capteurs d'essence sont exposés pour la première fois au MNBAQ en $2004^{\circ}$. Anne-Marie Ninacs, conservatrice et commissaire de l'exposition, s'appuie sur une documentation photographique pour installer l'œuvre dans la salle. Elle reprend la disposition faite par l'artiste lors des Jeux de la Francophonie à Gatineau, lorsqu'Annie Thibault a gagné le 1er prix en $2001^{\circ}$. Cette mise en vue avait été reprise quelques mois plus tard dans une exposition individuelle chez Pierre-François Ouellette art contemporain ". Pour le
8

Galerie d'art d'Ottawa, Ottawa, du 16 mai au 30 juin 1996.

9

Ils causent des systèmes. Acquisitions récentes en art actuel, Musée national des beaux-arts du Québec (Québec), 21 octobre 2004 au 29 août 2005. Commissaire: Anne-Marie Ninacs. 10 Installation sculpture. Capteurs d'essence (Jeux de la Francophonie), Galerie Montcalm-Maison du citoyen, Gatineau (Québec), du 14 au 24 juillet 2001.

11

Installation et photographies, PierreFrançois Ouellette Art contemporain, Montréal (Québec), du 20 octobre au 17 novembre 2001. 


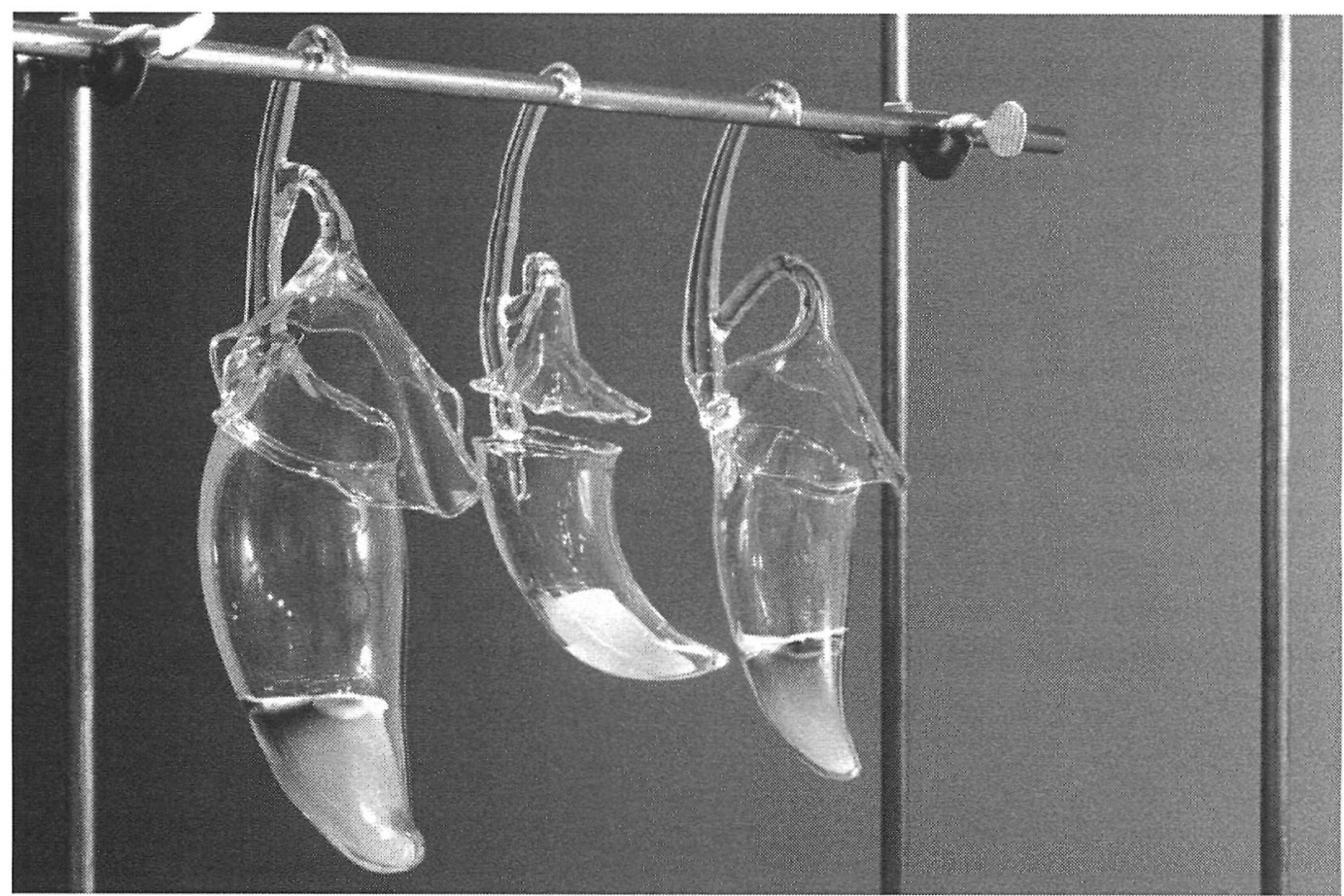

Illustrations 2 et 3

Annie Thibault (en collaboration avec Christophe Ciesla), Capteurs d'essence, 1997, version 2001, acier, verre soufflé, gélose et dépôt de la vie microscopique provenant de l'air ambiant (spores et bactéries), $250 \mathrm{x}$ $250 \times 250 \mathrm{~cm}$ (dimensions variables). Musée national des beaux-arts du Québec, 2005.2764. Achat pour la collection Prêt d'œuvres d'art en 2004, transfert à la collection du Musée national des beaux-arts du Québec. [Photos: Patrick Altman] 


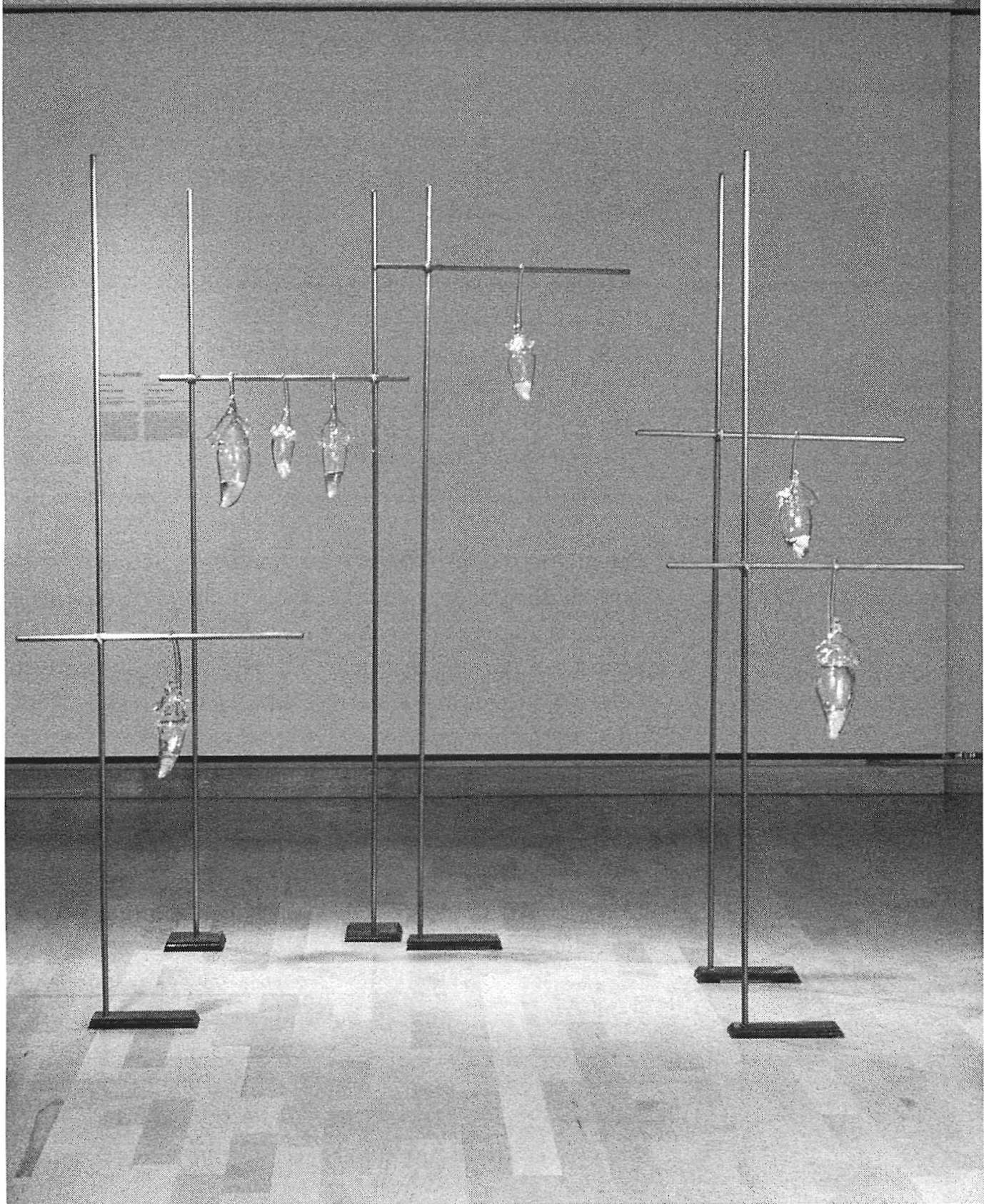


12

Pour les jeux de la Francophonie dans la salle d'exposition de la Galerie Montcalm-Maison du citoyen à Gatineau, les Capteurs d'essence étaient associés à une photographie de leur première mise en vue en extérieur en 1997 au Centre Art / Nature Boréal à la Minerve, Rivière Rouge (Québec). Dans la galerie montréalaise Pierre-François Ouellette Art contemporain, bien que l'exposition ne constituait pas une installation en elle-même, les Capteurs d'essence étaient entourés de photographies d'autres œuvres de l'artiste qui, placées à proximité, l'inscrivaient dans un contexte de référence. On reconnaît notamment une photographie du Cercle des sorcières qui avait déjà été associée aux Capteurs d'essence au Centre de sculpture Est-Nord-Est à Saint-JeanPort-Joli Québec), du 5 juillet au 3 septembre 1999.

13

Centre d'exposition du Vieux-

Palais, Saint-Jérôme (Québec), du 2 décembre 2001 au 3 février 2002. Commissaire: Andrée Matte.
MNBAQ, ces deux présentations en galerie sont devenues la référence pour la disposition des tiges en acier et la distribution des fioles sur les supports. Par contre, dans les salles du MNBAQ, Capteurs d'essence est isolée de La chambre des cultures, alors que dans les deux expositions de 2001 les autres œuvres qui l'entouraient l'y inscrivaient ${ }^{12}$. Ce n'est pas dans la mise en espace que cette œuvre garde sa variabilité. En effet, aucun document figurant au dossier de l'œuvre, qu'il soit rédigé par l'artiste ou un membre du musée, n'introduit la possibilité d'une variation du nombre de fioles pour l'exposition d'un fragment ou d'une adaptation à un lieu avec un accrochage extérieur dans des arbres par exemple. Cela ne semble pas avoir été évoqué lorsque l'œuvre est entrée dans la collection du MNBAQ. L'installation comprend sept fioles et les supports métalliques, et si ses dimensions sont approximatives dans la fiche technique, elles varient peu. En cela, Capteurs d'essence serait davantage à associer aux œuvres de la première tendance évoquée en introduction, celle où l'acquisition réduit la variation.

Cependant, Capteurs d'essence demande plus qu'une mise en espace. Elle doit être réactualisée à chaque fois qu'elle est montrée puisque les spores et les bactéries ambiantes doivent se nourrir sur place pour se développer dans les fioles. Cette contrainte avait déjà freiné la circulation de l'œuvre. En effet, les gagnants des Jeux de la Francophonie devaient participer à une exposition itinérante d'envergure, à laquelle Annie Thibault a dû renoncer parce qu'elle-même, ou une personne qu'elle aurait initiée, ne pouvait accompagner l'œuvre pour accomplir les gestes essentiels nécessaires. La délégation d'une partie de la production, alors problématique, s'est réglée avec la rédaction d'instructions écrites précises et la formation de personnes avant même que l'œuvre soit acquise. C'est ainsi qu'elle a participé en 2001 à l'exposition Reliques et reliquaires. Art religieux et art contemporain, à Saint-Jérôme, grâce à un tiers, sa galerie, qui a actualisé $l^{\prime} œ u v r e{ }^{13}$. Au MNBAQ, l'artiste délègue cette production à d'autres médiateurs formés, les techniciens du musée, encadrés par son protocole écrit, comme many things were left unsaid de Karilee Fuglem. Elle a, par ailleurs, équipé le musée d'instruments de laboratoire pour préparer la gélose 


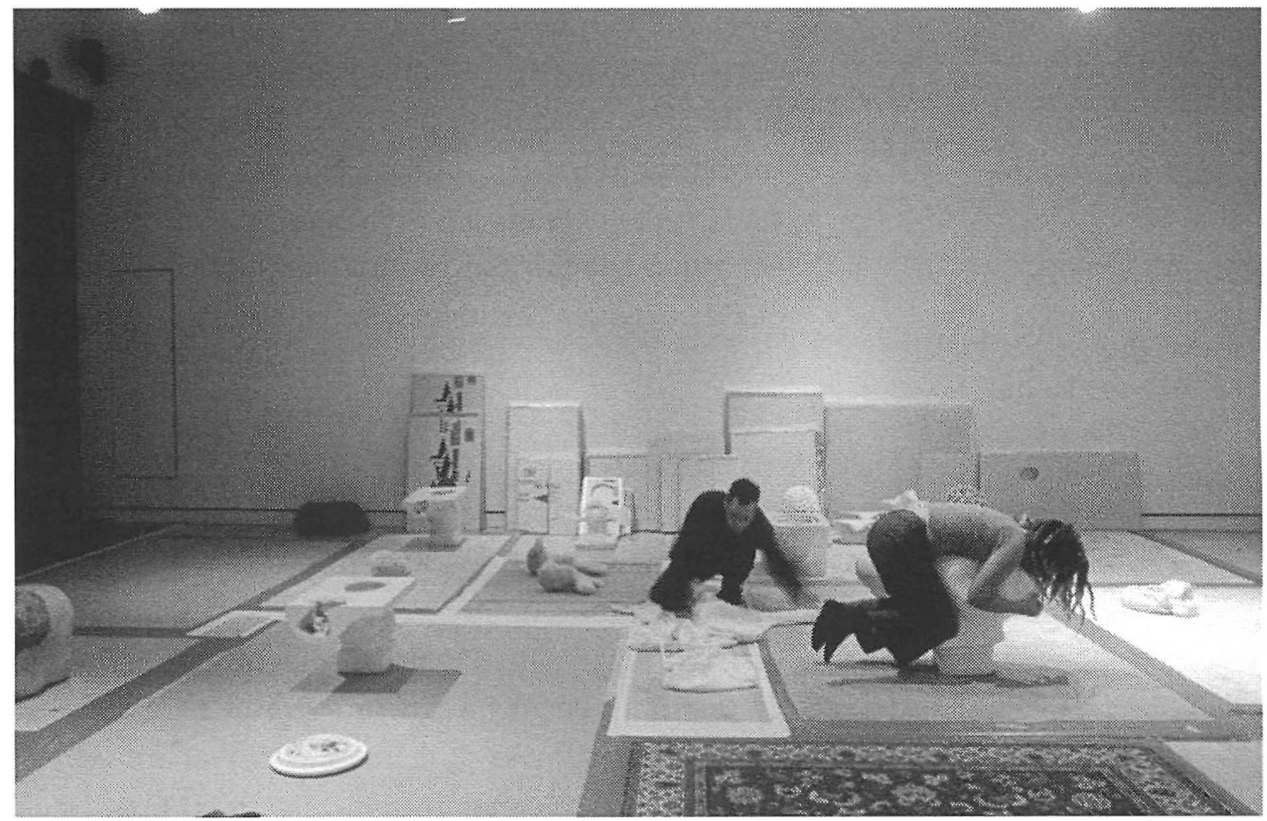

adéquatement et l'insérer dans les fioles (bouilloire, seringue, cuillère, balance, soucoupe et tasse à mesurer). Si l'adaptation disparaît, la variabilité inscrite au concept de l'œuvre, liée à la réactualisation pour le développement des cultures vivantes à chaque exposition, est conservée. Pour Capteurs d'essence, ce sont la documentation visuelle d'expositions antérieures en galeries, renforcée par les photographies prises dans les salles du MNBAO, plus l'apprentissage d'un savoir-faire guidé par les procédures de fabrication de la gélose et d'entretien des fioles rédigées par l'artiste qui assurent son intégrité et ses variations au sein du MNBAQ.

\section{Massimo GUERRERA, Darboral, 2000-2005, MNBAQ}

L'acquisition de Darboral a posé d'autres défis au MNBAQ qui souhaitait conserver son caractère évolutif dans la collection. Présenté par l'artiste comme une métaphore du corps humain, avec tout ce qui y transite et ce qui le transforme au gré des expériences, Darboral (ill. 4) est un espace de rencontre, où des personnes se croisent, échangent et côtoient des objets. Il s'inscrit dans ce qu'on nommera plus tard l'art relationnel, parmi des œuvres conçues en interaction avec le réel, engageant des échanges interhumains.

\section{Illustration 4}

Massimo Guerrera, Darboral, 20002005, polystyrène, tapis, rubans adhésif, acrylique, cire, gomme à mâcher et traces et matériaux divers, 148 x 970 x $44 \mathrm{~cm}$ (ensemble). Musée national des beaux-arts du Québec, Québec. Achat grâce à l'appui du Conseil des Arts du Canada dans le cadre de son programme d'aide aux acquisitions (2005-61.01 à 78). [Photo: Patrick Altman] 
14

Tout le temps / Every time, Biennale de Montréal, Centre international d'art contemporain, Montréal (Québec), du 28 septembre au 29 octobre 2000. Commissaire: Peggy Gale.
Avec Darboral, Massimo Guerrera, installé dans son atelier, dans une galerie, au musée ou sur la place publique, sollicite la participation du spectateur, convié en groupe ou individuellement. Chacun, par ses interventions, modifie la rencontre et, par conséquent, l'œuvre en cours. Ces échanges se matérialisent parfois dans des photographies, des dessins ou des objets, qui circulent et s'additionnent d'un événement à l'autre. Ils deviennent les traces des expériences des relations passées dans l'espace de Darboral.

L'œuvre est montrée pour la première fois en 2000, à la Biennale de Montréal ${ }^{14}$. L'artiste, présent ponctuellement, y invite un grand nombre de visiteurs à partager de la nourriture, transformant l'installation en fête. Les performances de Darboral peuvent aussi prendre une forme plus réduite avec des rencontres plus intimes dans l'atelier où les tapis de Darboral sont constamment disposés. Massimo Guerrera prolonge également ces rencontres seul, lorsqu'il dessine, photographie ou sculpte, en s'inspirant des relations qu'il a développées avec ses visiteurs. Les objets de Darboral assurent la pérennité de la part relationnelle de l'œuvre.

Pour chaque exposition, l'œuvre est activée par un repas en groupe, puis l'artiste y assure périodiquement une présence, souvent hebdomadaire, conduisant à des rencontres informelles avec les visiteurs. En l'absence du créateur, le spectateur parcourt une installation. Souvent baigné dans un environnement sonore, il se déplace sans soulier sur des tapis, parmi des traces de repas, de la nourriture, des meubles, des objets qu'il peut manipuler, des photographies qui montrent les actions passées, des dessins, des plantes, etc.

Massimo Guerrera poursuit Darboral avec cette double dimension performative et installative depuis 2000. C'est une œuvre évolutive qui, par conséquent, est variable d'une occurrence à l'autre. Elle s'adapte à l'espace physique, la plateforme de tapis de Darboral qui en circonscrit la superficie peut s'étendre à une galerie au complet ou, au contraire, se limiter à un seul tapis. Les objets qui la composent changent constamment, pouvant être ajoutés ou retirés de l'ensemble, de façon temporaire ou permanente, 
dans le cas d'une vente par exemple. Les objets subissent également des modifications au cours du temps, notamment en accumulant les traces de leurs usages. Plusieurs des objets ont été construits en collaboration avec le public et plusieurs ont circulé. L'artiste les confie à des personnes qui développent un rapport de proximité, puis ils sont rendus à l'artiste à l'occasion d'une nouvelle rencontre. L'objet dans Darboral semble dépasser le statut de la trace d'une action, il apparaît davantage comme un médiateur, essentiel dans son rôle avec le public, pour activer les mises en relation avec le contexte, le temps, l'espace et le corps'5. Les variations de Darboral sont également visibles par la multiplicité des titres employés par l'artiste, même si le mot au cœur du projet y apparaît souvent ${ }^{16}$. Aussi, retrouve-t-on dans l'installation des photographies qui, exposées sur les murs ou réunies en albums, témoignent du rôle de certains des éléments, tout en constituant une archive des actions posées dans l'œuvre.

En 2005, le MNBAQ acquiert 68 objets de Darboral par achat et par don. Retirés de l'ensemble pour l'acquisition, ils sont installés dans une nouvelle occurrence et constituent un second exemplaire de l'œuvre "7. Le choix des éléments, sélectionnés par l'artiste et la conservatrice Anne-Marie Ninacs, s'est fait en fonction de leur représentativité de l'ensemble ainsi que de ce qui pourrait être présenté dans les salles du musée ${ }^{18}$. L'acquisition se fait donc en vue d'une adaptation au MNBAQ. Dans la fiche d'acquisition, l'œuvre est décrite comme une "installation sculpturale " ${ }^{9}$. Ce faisant, on aurait pu fixer l'exemplaire afin de faire entrer une partie de cette œuvre évolutive dans le cadre connu des pratiques muséologiques. Mais ce n'était pas l'objectif du musée. Dès les premiers courriels échangés au sein de l'institution, on trouve l'idée formulée de reconstruire un atelier dans la salle d'exposition, autrement dit de présenter une œuvre en cours qui se transforme au fur et à mesure des rencontres, un espace dans lequel la production se poursuit.

On pourrait penser que l'acquisition a fixé le nombre d'éléments de cet exemplaire de Darboral, mais ce n'est pas le
15

Pour un développement sur le statut des objets dans Darboral, voir FRASER Marie et Marie-Josée LAFORTUNE. "Massimo Guerrera". In. Gestes d'artistes. Montréal: Optica, 2003, p. 42-49. 16

A titre exemplaire, Massimo Guerrera donne différents noms à son exposition: Un trait d'union entre le visible et l'invisible (Darboral) / A Hyphen between the Visible and the Invisible (Darboral) lorsqu'elle est présentée à la Fonderie Darling, Montréal (Québec) du 26 juin au 31 août 2008; Darboral (Et l'entretien patient d'un champ de pratique) dans le cadre de l'exposition intitulée Massimo Guerrera. Darboral à la Galerie Joyce Yahouda, Montréal, du 21 avril au 20 mai 2006 ; et Darboral (phase III), la performance présentée au Festival international de nouvelle danse de Montréal Salle Beverly Webster, Musée d'art contemporain de Montréal, Montréal (Québec), du 2 au 6 octobre 2001. 17

Il existe à ce jour trois exemplaires de Darboral: celui de l'artiste qu'il poursuit, celui du MNBAQ que nous analysons ( $\left.n^{2} 2005-61\right)$ et un autre qui a été acquis à l'hiver 2009 par le MBAC ( $\left.n^{2} 42535.1-16\right)$ à la suite de l'exposition Flagrant délit. La performance du spectateur, Musée des beaux-arts du Canada, Ottawa (Ont.), du 17 octobre 2008 au 15 février 2009. Ce troisième exemplaire a également été exposé dans L'Espace Shawinigan, Cité de l'énergie, Shawinigan (Québec), du 20 juin au 27 septembre 2009 Commissaire: Josée Drouin-Brisebois Nous n'analyserons pas ce troisième exemplaire ici, car nous n'avons pu rencontrer tous les intervenants liés à cette acquisition avant de terminer notre article.

18

Courriel d'Anne-Marie Ninacs à

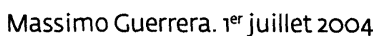
Musée national des beaux-arts du Québec, Québec, dossier 2005-61-01-42.

19

Fiche d'acquisition. Musée nationa des beaux-arts du Québec, Québec, dossier 2005-61-01-42. 
20

NINACS, Anne-Marie. "Consignes pour la conservation et l'exposition de Darboral de Massimo Guerrera à la suite de discussions avec l'artiste de novembre 2004 à avril $2005 "$, $5 \mathrm{p}$. Musée national des beauxarts du Québec, Québec, dossier 2005-61-01-42.

21

La préservation des traces des usages sur les objets se retrouve également dans BELLEAU, Claude. Rapport d'état de conservation. 8 juillet 2005. Musée national des beaux-arts du Québec, Québec, dossier 2005-61-01-42.

22

Des fragments de Darboral ont été intégrés dans la salle 2 de la collection permanente du MNBAQ intitulée: "Figuration et abstraction au Québec, 1940-1960", pour l'exposition Intrus/Intruder (op.cit.). Par ailleurs, le MNBAQ a prêté un objet au MBAC afin qu'il soit intégré dans une nouvelle occurrence de Darboral pour l'exposition Flagrant délit. La performance du spectateur. au Musée des beaux-arts du Canada, Ottawa (Ontario), du 17 octobre 2008 au 15 février 2009; cette exposition itinérante a aussi été présentée à L'Espace Shawinigan, Cité de l'énergie, Shawinigan (Québec), du 20 juin au 27 septembre 2009. Commissaire: Josée Drouin-Brisebois

23

Darboral, ici, maintenant, avec l'impermanence de nos restes (phase III), Musée national des beaux-arts du Québec (Québec), du 14 novembre 2002 au 12 janvier 2003. Commissaire: Anne-Marie Ninacs, alors contractuelle au musée et membre du Comité d'acquisition. Le rôle de référence de cette exposition est encore plus clair quand on constate que c'est cet environnement sonore qui a été intégré à l'acquisition alors que plusieurs ont été produits au cours de la vie de Darboral. cas, comme on peut le remarquer à la lecture du document de cinq pages produit par Anne-Marie Ninacs pour accompagner l'œuvre, rédigé à la suite de discussions avec l'artiste qui se sont déroulées de novembre 2004 à avril $2005^{20}$. Pour ne souligner que ce qui concerne nos préoccupations sur les variations, elle y aborde la possibilité d'ajouts d'autres objets au corpus de cet exemplaire grâce à des dons subséquents de collectionneurs ainsi que les modifications subies par les objets à préserver. Elle parle de la patine à conserver car elle est signifiante ${ }^{21}$, elle mentionne la possibilité que l'artiste lui-même y apporte des changements dans une réanimation de l'œuvre ou encore que les éléments vivent des temporalités différentes par leur circulation, en tant que fragments de l'ensemble, lorsqu'ils sont joints à d'autres expositions comme ce fut le cas pour Intrus/Intruders et Flagrant délit. La performance du spectateur ${ }^{22}$. Anne-Marie Ninacs développe aussi sur l'installation de Darboral en s'appuyant sur des photographies d'une occurrence antérieure, celle présentée au MNBAQ mise en place par l'artiste ${ }^{23}$, qui fait office de référence pour l'avenir. Elle précise également le type de nourriture à acheter et son renouvellement. Enfin, elle envisage trois modes de présentation de ce corpus: l'ensemble, la version réduite et le fragment.

À la lecture du document nous comprenons qu'il est prévu que les objets varient. En dehors de ses composantes physiques, l'aspect performatif de Darboral a également été préservé. Tout d'abord, les consignes laissent une grande ouverture à la participation de l'artiste dans l'exemplaire du MNBAQ, même s'il est précisé que "sa présence n'est pas requise pour son bon fonctionnement". Il est prévu que l'œuvre soit activée par un repas collectif de dix à vingt personnes pour lesquelles ce projet est signifiant. Les traces de ce repas, qui doivent être laissées sur place, feront partie des éléments de l'installation. On souhaite que Darboral demeure une œuvre relationnelle directe pour laquelle l'aspect de coproduction dans le présent est très important. Cependant, deux usages performatifs essentiels de Darboral sont altérés avec l'acquisition. Tout d'abord, la manipulation des objets pendant l'exposition, faite par les visiteurs 
sur la plateforme. Afin de remédier à cette situation, AnneMarie Ninacs envisage qu'en présence de l'artiste le public puisse toucher les objets et elle prévoit aussi des séances, sous la supervision "d'une personne formée à l'esprit du projet par le Musée", où les visiteurs seraient invités à manipuler les objets. En ce qui concerne l'échange, incompatible avec les normes muséologiques, il disparaît complètement du projet. L'exemplaire du musée garde malgré tout une trace de cet aspect de l'œuvre, basé sur l'échange nominatif, actif et volontaire, avec la liste des noms des participants au projet dans sa fiche d'acquisition.

Au MNBAQ, les conditions fixées dans le document, mais qui n'ont pas encore été mises en application car l'ensemble n'a pas été réexposé, signifient le désir que Darboral continue de varier au sein de la collection, qu'on montre quelque chose en cours. Les idées au centre de cette œuvre évolutive sont la circulation, la rencontre et l'échange, difficiles à entrer dans une collection et à garder actives ${ }^{24}$. La délégation de la production de l'œuvre au musée, discutée pendant six mois et fixée dans le document, est encore plus large que dans les cas de Karilee Fuglem et d'Annie Thibault. Les termes rédigés par la conservatrice tentent de conserver l'intégrité matérielle et conceptuelle de Darboral, mais, comme nous l'avons vu, une partie est malgré tout altérée.

Définir de telles conditions, afin de maintenir la variabilité des œuvres, n'est pas une procédure familière au MNBAQ. Elles apparaissent clairement liées à la présence de la conservatrice de l'art actuel du musée, en poste de 2002 à 2006. Anne-Marie Ninacs a transposé au musée les questions explorées dans sa pratique de commissariat ${ }^{25}$. Celle-ci est préoccupée par la présence des pratiques d'art relationnel au sein des collections et refuse que le musée ne s'en tienne qu'à des dessins ou des documents pour en rendre compte. Sa compréhension des œuvres et de son rôle de conservatrice lui permet "de sensibiliser la direction du MNBAQ au défi que représentaient ces nouvelles pratiques et à l'importance de le relever ici et maintenant [...] et de sensibiliser les artistes, un à un, en leur posant la question de l'acquisition alors que plusieurs s'excluaient
24

Le 12 février 2009, à notre question qu'est-ce que Darboral? Massimo Guerrera a répondu: "Darboral, c'est ça " en parlant de notre discussion. "Ce n'est pas les objets. Les objets sont des réflecteurs de la parole. Darboral est immatériel. " Dans sa perception de son œuvre évolutive, quatre ans après son acquisition, l'artiste met de plus en plus l'accent sur le "performatif". (Entrevue non publiée.)

25

Anne-Marie NINACS a exprimé son point de vue sur la position du commissaire dans un article: "Signer ou s'effacer? Pour une pratique éthique du commissariat d'exposition". Esse, $n^{2}$ 57, mai 2006, p. 6-10, version abrégée d'une conférence prononcée à la table-ronde Unspoken Assumptions: Visual Art Curators in Context. Banff Centre, Banff (Alberta), le 16 juillet 2005. (NINACS, Anne-Marie. Towards More Ethical Curatorial Practices. <http://Curatorsincontext.ca/transcripts/ninacs. pdf> (consulté le 27 juillet 2010).) 
26

NINACS, Anne-Marie. "Conserver l'impermanence. L'acquisition des œuvres de Massimo Guerrera pour une collection muséale". Colloque La réexposition de l'art contemporain : problèmes et enjeux de la préservation des œuvres. ACFAS, 14 mai 2004, transcription de communication. Musée national des beaux-arts du Québec, Québec, dossier 2005-61-01-42. 27

II s'agit du projet La constitution du patrimoine culturel : questions et débats autour de la préservation des cuvres d'art récent, sous la direction de Jan Marontate (Acadia University, Wolfville, Nouvelle-Écosse), 20042005, subventionné par le CRSH.

28

Sur l'origine et les débuts du Mamco, voir l'entrevue de Christian Bernard par Jean-Yves JOUANNAIS. "Mamco. Point de vue et ambition de son responsable. Christian Bernard ". Art Press, n² 194, septembre 1994, P. IV-VI.

29

Pour le détail des objectifs du Mamco, voir MAMCO. Qu'est-ce que le Mamco ? <http://www.mamco.ch/ musee.html> (consulté le 26 juillet 2010). d'emblée du musée " ${ }^{26}$. En collaborant étroitement avec les artistes, que ce soit Karilee Fuglem, Annie Thibault ou Massimo Guerrera, Anne-Marie Ninacs installe au sein du MNBAQ de nouvelles pratiques de conservation, avec des œuvres variables afin que le spectateur puisse expérimenter l'œuvre plutôt que se retrouver devant un témoignage. Pour appuyer sa réflexion, lors de processus d'acquisition de Darboral, elle n'a pas hésité à collaborer à un groupe de recherche sur la réexposition des œuvres ${ }^{27}$.

\section{La production ininterrompue: l'atelier et l'artiste dans le musée}

\section{SARKIS, L'Atelier depuis 19380, en place depuis 1994, Mamco}

Cette proximité d'un conservateur avec les œuvres qui engage l'institution muséale à élargir ses modalités de collections n'est pas exclusive au Québec. Pour mieux rendre compte de la singularité de L'Atelier depuis 19380 de Sarkis dans le milieu muséologique, il est préférable d'expliquer avant tout en quoi le Mamco est un musée atypique. Fondé en 1994 dans un ancien édifice industriel, le Musée d'art moderne et contemporain de Genève est une création de Christian Bernard ${ }^{28}$. Deux idées essentielles liées à sa fondation sont à l'origine de l'exposition de l'œuvre de Sarkis : la volonté d'une "variation des types d'espaces et d'accrochages évoquant l'histoire du musée et des styles d'exposition (de l'appartement du collectionneur au plateau brut du loft, en passant par la boîte blanche ('white cube'), la cellule, l'atelier, l'entrepôt, etc.)", ainsi que "l'insistance sur les espaces dévolus à des présentations monographiques durables [...] [avec la] gestion évolutive, par les artistes eux-mêmes, de leurs espaces monographiques "29. Le directeur souhaite créer un musée différent à cause du décalage qu'il a remarqué entre les pratiques artistiques depuis les années 1960 et l'offre muséographique. Par conséquent, pour un musée d'art contemporain qui travaille avec des artistes vivants, Christian Bernard pense à une institution expérimentale qui juxtaposerait au sein d'un même lieu 
différents modes d'accrochage. Il réfère ainsi à l'histoire des musées et à l'histoire de l'art, proposant "un outil de réflexion critique sur l'art contemporain et sur l'institution qui a en charge de le faire connaître et de le conserver ${ }^{3 \circ}$. Pour ce faire, il fait appel à son vaste réseau d'artistes et de collectionneurs afin de constituer la collection du Mamco ${ }^{31}$. Celle-ci n'est pas pour autant immobile dans les salles. Les espaces monographiques évoluent selon des temporalités différentes, si bien que le musée fait l'objet de mouvements constants, sans compter que les espaces temporaires et permanents se jouxtent dans l'institution ${ }^{32}$. C'est dans ce cadre que Christian Bernard engage une discussion avec Sarkis.

Si le directeur fait appel à Sarkis, c'est notamment à cause du rôle particulier de son atelier, bien avant L'Atelier depuis 19380. Selon le directeur, "le lieu de son œuvre, c'est son atelier. C'est là où la totalité des éléments qui constituent le noyau dur de son œuvre se tient. Ses expositions ne sont jamais que des dilatations, parfois géniales, parfois décevantes, de ce noyau ${ }^{33}$." Si les installations s'adaptent et développent des liens étroits avec leur espace d'exposition temporaire que l'artiste transforme brièvement en atelier, elles s'insèrent malgré tout dans une continuité. En dehors de l'exposition temporaire, elles s'inscrivent dans la durée au sein de l'œuvre du créateur. Sorties de l'atelier pour être montrées en public, les œuvres y retournent une fois l'exposition terminée. Leur vie se poursuit en prenant place dans un immense assemblage, développant des liens, des échanges avec les autres œuvres et avec les objets qui y sont amassés. En cela, Sarkis s'inscrit dans des pratiques artistiques où l'atelier prolonge l'œuvre, tels ceux de Constantin Brancusi, Jean Gorin, Félix del Marle, Piet Mondrian, Kurt Schwitters, etc.

Plus précisément, la pratique d'atelier de Sarkis se rapproche du modèle du laboratoire scientifique ${ }^{34}$. Dans ces deux lieux privés où seuls quelques initiés pénètrent, on expérimente des idées et on déploie imagination et créativité sans entrave avant de divulguer les résultats à l'extérieur. Et parce que le laboratoire et l'atelier sont des lieux
30

BERNARD, Christian. "Plongez dans le Mamco pour nager dans l'art contemporain". Tribune de Genève, $\jmath^{\text {er }}$ décembre 1994.

31

La collection du Mamco se compose d'œuvres en dépôt à long terme ainsi que du fonds de l'Association pour un musée d'art moderne et d'autres œuvres acquises depuis son ouverture.

32

Dans une des ses allocutions, Christian Bernard explique les mouvements de la collection. ("Un musée en construction. Conférence au Centre Pompidou (novembre 2003)". Publié dans propos du Mamco. Christian Bernard. Interviews. Déclarations. Conférences. 1994-2008. Revue de presse du Mamco.)

33

GAUVILLE, Hervé et Elisabeth LEBOVICI. "Un musée est une maison de rendez-vous". Libération, 17 janvier 2004, p. 50.

34

Dans notre recherche doctorale (RODRICUEZ, op.cit., p. 215-239), nous avons déjà analysé des modèles d'ateliers, dont celui du laboratoire, à partir de l'exemple de ceux de Piet Mondrian, en nous appuyant sur des analyses ethnographiques (depuis les années 1970) qui étudient la communauté scientifique dans son milieu et révèlent le laboratoire comme un des lieux de l'activité et du réseau scientifique. 
35

LATOUR, Bruno et Steve WOOGLAR. La Vie de laboratoire. La Production des faits scientifiques. Traduit de I'anglais par BIEZUNSKI, Michel. Paris: La Découverte (Poche), 1996 (c. 1979), p. 84.

36

Ibid., p. 8 et 13; LATOUR, Bruno, La Science en action. Introduction à la sociologie des sciences. Traduit de l'anglais par BIEZUNSKI, Michel. Paris, Gallimard (Folio), 1995 (c. 1987), p. 155.

37

Pour une analyse des éléments clés de l'œuvre de Sarkis que sont la mise en scène, le temps, la lumière et la mémoire, voir ZABUNYAN, Elvan. "Illumination". In. Sarkis. Scènes de nuit. Scènes de jour. Grenoble: Le Magasin. Centre d'art contemporain de Grenoble, 1991,

p. 7-15.

38

Nonobstant ce rapprochement de l'atelier de Sarkis et du laboratoire scientifique, il ne faudrait pas confondre les deux espaces. L'atelier est beaucoup moins formel et codifié que le laboratoire et surtout moins hiérarchisé. Sarkis y travaille seul et ses méthodes sont différentes de celles des scientifiques. Leur finalité les sépare. En effet, dans le laboratoire, le scientifique effectue un contrôle du lieu afin qu'il interfère le moins possible dans les conditions d'expérimentation. Au contraire, dans l'atelier de Sarkis, le lieu est signifiant pour l'œuvre.

39

Cette expression "atelier de voyage» se retrouve dès les débuts du Mamco. Christian BERNARD l'emploie en 1994 dans son entrevue avec JeanYves Jouannais (" Mamco. Point de vue et ambition de son responsable. Christian Bernard ". Art Press, n 194. septembre 1994, p. VI) et il la reprend lors de notre rencontre. (Entrevue non publiée avec Christian Bernard, le 18 juin 2009.)

40

DUMONT, Étienne. "Les antihéros de Christian Bernard ". Tribune de Genève, 21 septembre 1994. de référence, ils deviennent des espaces susceptibles de contribuer à la compréhension du travail. Le laboratoire construit les expériences à venir par son activité en cours et s'appuie sur la synthèse des données antérieures validées par diverses controverses ${ }^{35}$. L'atelier de Sarkis, à Villejuif, semble également doué de parole. Il distribue des indices sur sa démarche artistique parce qu'il renferme différents objets, livres, instruments, œuvres antérieures, œuvres à différents stades, etc. qui témoignent et participent au cheminement créatif. Ils partagent encore une autre caractéristique : la lumière. Dans les écrits de Bruno Latour, le laboratoire semble toujours brillamment éclairé ${ }^{36}$. Or, le rôle de la lumière dans l'œuvre de Sarkis est fondamental ${ }^{37}$.

Lieu permanent d'élaboration et de mise à l'épreuve, l'atelier de Sarkis à Villejuif ressemble à un laboratoire scientifique, une structure fixe dans laquelle il revient constamment travailler, contenant par essence toutes les possibilités ${ }^{38}$. C'est le lieu d'origine de son projet artistique, il est organisé, réfléchi, mis en scène, aménagé et modifié selon l'évolution de sa pratique et de ses projets d'exposition. L'atelier, espace idéal de présentation, apparaît alors comme le prolongement expérimental de l'œuvre et la condition de son accomplissement. Il acquiert ainsi une fonction essentielle dans le processus de création tout entier sur laquelle Sarkis s'est souvent exprimé dans ses entretiens. C'est dans ce contexte que Christian Bernard lui offre un moyen de production unique et que l'artiste installe "un atelier de voyage ${ }^{39}$ dans le Mamco.

L'Atelier depuis 19380 (ill. 5) est indissociable du Mamco. Tout d'abord dans son concept, avec l'idée d'exposer l'atelier comme un des lieux de l'art adoptés par les artistes, in progress, où la production se perpétue. D'un point de vue physique, c'est également le directeur du musée qui a imaginé la division des plans libres de l'édifice industriel en cellules modulables, après une visite des fresques de Fra Angelico au couvent San Marco à Florence ${ }^{40}$. Sarkis a toutefois restreint la taille de l'espace qui lui était réservé afin de l'isoler des autres par des couloirs latéraux ${ }^{41}$. De l'extérieur, 


\section{Illustration 5}

Sarkis, L'atelier depuis 19380, 19942010, cabane-atelier conçue par l'artiste, collection Mamco, Genève. [Photo: I. Kalkkinen]

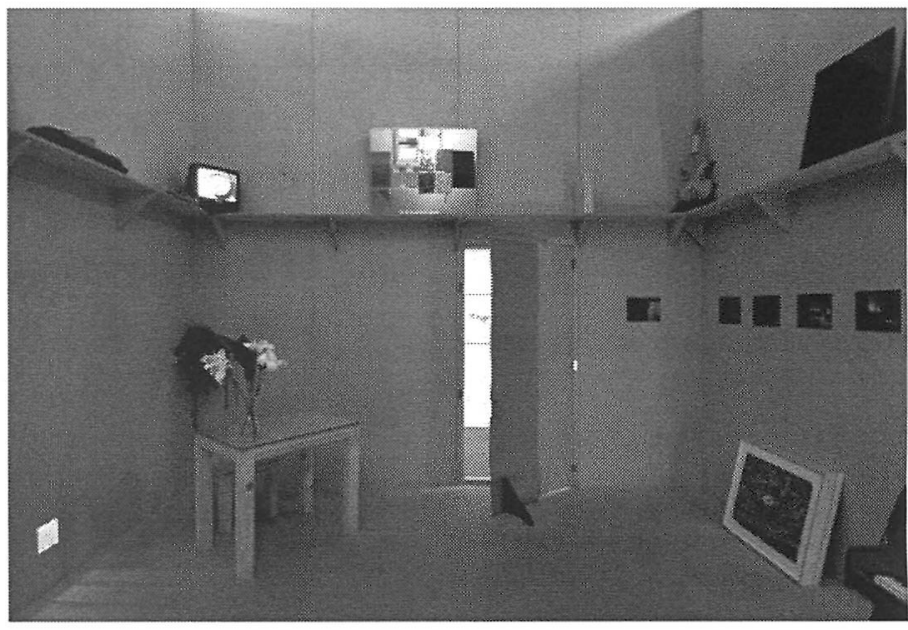

l'atelier paraît indépendant des autres lieux ${ }^{42}$. Enfin, le maigre budget de lancement du musée a incité à choisir le bois aggloméré comme matériau pour construire la structure modulable des cellules. C'est également en bois que Sarkis ferme son espace avec une cloison, une porte et un seuil qu'il faut enjamber pour s'y introduire, et qu'il fait construire du mobilier (tabouret, table et tablette). Les murs et le plancher en bois sont laissés nus. Il refuse l'éclairage d'origine de l'édifice et fait percer une fenêtre, orientée à l'ouest, par laquelle la lumière naturelle pénètre chaque après-midi à travers un vitrail et balaie l'atelier. La lumière artificielle est pourtant présente par le rayonnement des œuvres exposées dans l'espace, notamment le fluorescent rouge d'un exemplaire de la série 19380, qui a été construit exclusivement pour le Mamco. La variation de son intensité varie, elle est fixée sur la respiration de l'artiste. En plus des constructions spécifiques à ce lieu, l'artiste l'investit en y apportant des objets de son atelier parisien. Christian Bernard s'adresse aussi à des collectionneurs privés suisses pour qu'ils y déposent leurs œuvres. En cela, L'Atelier depuis 19380 fait figure d'œuvre-synthèse, c'està-dire qu'il réunit des œuvres antérieures dans une nouvelle présentation. Cependant, l'objectif n'est pas d'en faire une rétrospective, mais bien un atelier selon la conception du travail de Sarkis, un lieu unique qui participe à la mise en contexte de l'œuvre, où l'artiste crée des résonnances.
41

Nous remercions sincèrement Sophie Costes de nous avoir accompagnée dans une visite guidée de l'atelier ainsi que Christian Bernard qui nous a permis de consulter ses archives.

42

Cette impression s'est accentuée au cours du temps car, lorsque nous avons visité le musée en 2009 , L'Atelier depuis 19380 était le seul espace en bois qui restait exposé dans le Mamco. Ses matériaux et sa couleur tranchent considérablement sur les autres espaces. 
43

Parmi les œuvres particulières en dépôt au Mamco qui ont figuré dans l'atelier, mentionnons Drakula, 1979: La perruque en bande magnétique sur la tête en terre glaise de Sarkis, 1961-1979-1980; KK, 1980; Viola d'amour du Capt. Sarkis, 1985; Conversation entre J.M. Hauer et A. Schönberg, 1983; Kriegsschatz Leidschatz, 1990; Ma chambre de la rue Krutenau en satellite, 1990.

44

Ces sous-titres de L'Atelier depuis 19380 apparaissent depuis le 8 état, en 2000 , lorsque l'écriture fait son apparition dans l'atelier. Ils sont également très souvent les titres des aquarelles que l'artiste a réalisées sur place, dont: L'Écho de ma main, $8^{e}$ état; Le cri XX, $9^{e}$ état; Je tombe la couleur jaune profonde, $10^{\mathrm{e}}$ état. 45

Lorsque nous avons visité l'atelier en juin 2009, les pétales étaient regroupés au sol, près de l'œuvre Kriegsschatz Leidschatz, et des pétales rouges en faisaient partie.

46

Etant donné les rôles de la photographie et de la lumière dans l'œuvre de Sarkis, le geste de Sophie Costes est signifiant dans L'Atelier depuis 19380.
Une ou deux fois par année, Sarkis se rend dans son atelier du Mamco pour y travailler. Son séjour commence par une visite critique du musée avant de se réfugier dans son espace jusqu'au soir. La porte, entrebâillée ou poussée, n'est pas fermée aux visiteurs. Cependant, Sarkis ne donne pas de rendez-vous au public comme Massimo Guerrera dans les expositions de Darboral. L'objectif de l'œuvre est différent. Sarkis ne crée pas une œuvre en interaction avec le public. Il s'installe dans son atelier, un lieu privé, entouré de ses œuvres, où il se concentre sur la création et non sa relation avec autrui. Les inventaires de l'atelier du Mamco ainsi que les photographies périodiques du lieu montrent la variété de ses gestes au cours des années. Son travail d'aquarelle y est néanmoins récurrent. Les œuvres présentes dans L'Atelier depuis 19380 changent également, que ce soit parce que l'artiste modifie leur assemblage ou parce qu'elles sont retirées pour participer à des expositions temporaires extérieures, les œuvres en dépôt par exemple ${ }^{43}$. Mis à part la documentation photographique et les plans successifs de l'espace, la numérotation des états indique le nombre de variations, sans compter que parfois Sarkis a ajouté un sous-titre à ses interventions ${ }^{44}$.

En dehors des présences ponctuelles de l'artiste, L'Atelier depuis 19380 varie également parce que l'artiste délègue des gestes. En plus de préparer le matériel nécessaire à la venue de l'artiste, Sophie Costes, conservatrice de la collection du Mamco, entretient quotidiennement l'œuvre en posant par exemple une goutte de parfum Vol de nuit de Guerlain sur une pyramide de talc. Depuis son entrée en poste en 1995, la conservatrice s'occupe de cette œuvre, se décrivant avec humour comme sa pythie. Sophie Costes a développé une connaissance approfondie de l'œuvre de Sarkis et progressivement en est venue à poser des gestes dans L'Atelier depuis 19380. Par exemple, elle a recueilli les pétales des roses lors $\mathrm{du}$ vernissage d'une exposition de Sarkis à Istanbul et les a déposés sur la table dans l'atelier ${ }^{45}$; elle a photographié la lumière dans l'atelier ${ }^{46}$; le jour de la Sainte-Sophie, elle y dépose un bouquet de fleurs blanches sur l'étagère devant l'œuvre À partir du miroir de Sainte-Sophie (1998); etc. Tous ces gestes tissent des liens entre les œuvres de Sarkis, 
ils poursuivent les rencontres en ajoutant à la mémoire de l'œuvre. En dehors du personnel institutionnel, d'autres personnes posent des gestes, comme Mme Selvi, collectionneuse suisse de Sarkis qui a déposé ses œuvres dans l'espace et y a fait livrer des fleurs coupées, présentées dans un vase posé sur la table, pendant sept ans. L'Atelier depuis 19380 est en constante évolution, sous l'autorité de Sarkis, avec des gestes et des présences quotidiennes qui ajoutent à la vie de l'atelier.

Grâce à la présence de l'artiste et à la délégation, l'atelier du Mamco vit une dynamique de production, tout comme son pendant parisien. Cependant, il ne nous semble pas que L'Atelier depuis 19380 relève du modèle du laboratoire, comme l'atelier de Villejuif, mais plutôt de celui de l'atelier-œuvre d'art ${ }^{47}$. Les deux espaces sont différents. Dans L'Atelier depuis 19380. Blackout, 1977-2009, 22e état que nous avons $\mathrm{vu}$, par exemple, nous ne retrouvons pas d'outils traditionnels, comme les pinceaux pour les aquarelles, ni d'œuvres en cours de création pour une exposition à venir, comme dans l'autre espace. Ce que Sarkis conserve de l'atelier c'est la production périodique sur place et le processus évolutif d'assemblage et de sédimentation de la mémoire si caractéristiques de son œuvre. En cela, l'atelier du Mamco se rapproche de celui de Constantin Brancusi dans lequel l'artiste est maître du temps et de l'espace, où il contrôle les paramètres de présentation tels la lumière, le voisinage, la proximité des œuvres, les échos formels et conceptuels, les couleurs, le son, le parcours du spectateur, etc. qui influencent la perception. Au Mamco, l'atelier-œuvre donne une dimension supplémentaire à ce qu'il présente, créant des rapports nés de leurs juxtapositions. L'atelier mis en scène s'expose, il reçoit des visiteurs. Les spectateurs évaluent l'ensemble dans un espace précis, ordonné en installation qui fait œuvre. L'espace intervient à titre d'élément plastique, un liant actif et pas seulement un contenant. De plus, cet atelier-œuvre porte un titre, il est collectionné et exposé en permanence au musée ${ }^{48}$. La présentation des installations dans cet atelier-œuvre complète leur compréhension. En outre, l'atelier-œuvre déploie un
47

Nous avons pu discuter de cette idée avec Sarkis qui appuie notre point de vue. (Entrevue non publiée avec Sarkis dans son atelier à Villejuif, le 23 juin 2009.) Dans notre recherche doctorale (RODRIGUEZ, op. cit., p. 240-260), nous avons déjà analysé ce modèle d'atelier-œuvre d'art, à partir de l'exemple de Constantin Brancusi.

48

Depuis son installation en 1994. L'Atelier depuis 19380 n'a été démonté qu'une seule fois pour les besoins d'une exposition temporaire. Il est constamment exposé au Mamco. Il a été envisagé de l'exposer au Musée national d'art moderne à Paris, mais ce projet ne s'est pas concrétisé. Ces éléments ramènent aux conditions d'attribution du statut d'œuvre d'art d'un point de vue sociologique. (Voir HEINICH, Nathalie. "Les objets-personnes: fétiches, reliques et œuvres d'art". Sociologie de l'art, $n^{2} 6,1993$, p. 36.) 
49

Sarkis s'est exprimé à plusieurs reprises sur la nécessité de faire vivre les œuvres, même après la disparition de l'artiste. Citons, entre autres: DUCROS, Françoise. "La parole des choses". Galeries Magazine, $n^{\circ}$ 60, été 1994, p. 43-47, 98; et SARKIS. "Sarkis". Conservation et restauration des œuvres d'art contemporain. Paris: La documentation française, 1994, p. 48-51. projet artistique où tout est en étroit rapport avec Sarkis et se présente comme une mise en abîme d'une exposition.

Seulement, contrairement à l'atelier de Brancusi, L'Atelier depuis 19380 n'est pas figé. La lumière change, des parfums flottent, 19380 respire, le plancher craque et porte les traces des passages tout comme les trous dans les murs faits pour les accrochages antérieurs. La question cruciale qu'il pose concerne bien entendu sa pérennité dans la production. Rien n'apparaît dans le dossier de l'œuvre sur ce sujet. Depuis 2007, l'artiste et le directeur du musée en discutent et, peu à peu, des possibilités sont envisagées afin de la poursuivre. Par exemple, lorsque l'artiste vient au Mamco, il réalise plusieurs aquarelles de petit format, mais une seule est accrochée au mur sous le vitrail. Le nombre d'aquarelles est associé à l'âge de Sarkis et le Mamco en dispose de quelques-unes d'avance pour poursuivre cet accrochage annuel. Sarkis, qui a déjà mesuré l'écart entre les œuvres de Joseph Beuys et de Marcel Broodthaers, par exemple, et ce que l'on en trouve dans les musées, a mentionné à plusieurs reprises un métier qui devrait être créé en art contemporain, celui "d'interprétateur d'œuvres"49. Sarkis dit de lui-même qu'il interprète l'œuvre lorsqu'il la met en espace dans un lieu d'exposition, comme le mentionne également Christian Bernard. L'interprète, en fonction de sa propre histoire et de celle de l'objet, met en scène l'œuvre d'art. Mais l'interprète de L'Atelier depuis 19380 n'a pas encore été choisi et beaucoup de décisions restent à prendre quant à sa pérennité, du fait que son intégrité repose notamment sur une œuvre vivante en résonance avec le visiteur.

\section{Conclusion : l'œuvre variable collectionnée ou l'intention de l'artiste en valeur culte}

A partir d'un corpus d'œuvres qui appartiennent à des collections muséales, nous avons étudié comment l'intégrité d'une œuvre variable était préservée au musée lorsque la production se poursuit, qu'elle soit déléguée aux professionnels du musée (Fuglem, Cuerrera, Thibault) ou, plus exceptionnellement, continuée par l'artiste (Sarkis). 
Nos analyses ont montré la singularité de chaque œuvre étudiée. Chacune requiert des décisions particulières, même si le mode de la variabilité est identique. Nous avons ainsi analysé plusieurs œuvres qui varient par l'adaptation (Fuglem, Thibault), la réactualisation (Fuglem, Thibault), le fragment (Guerrera, Thibault) et l'évolution (Guerrera, Sarkis). Divers cas de figure apparaissent au sein de la même institution, ce qui marque davantage la singularité de chaque œuvre à cause de la diversité des pratiques artistiques. Le processus d'acquisition apparaît clairement comme un moment charnière dans la vie de l'œuvre.

En ce qui concerne les médiateurs en action, les études de cas témoignent du processus collectif de l'acquisition (artiste, conservateur, restaurateur, technicien) pour définir en collaboration les conditions qui assureront l'intégrité de l'œuvre, transformant le musée en atelier et le personnel du musée en assistants de l'artiste ${ }^{50}$. Cette coopération se poursuit avec la production collective et multidisciplinaire de la documentation nécessaire aux réexpositions. L'artiste, mais aussi les techniciens, le restaurateur et le conservateur, rédigent, dessinent, photographient, cartographient, enregistrent des mots, des gestes, des objets afin de circonscrire l'œuvre dans ses différentes dimensions matérielles, esthétiques et conceptuelles. Ils sont produits pour l'acquisition et se multiplient au fur et à mesure que l'œuvre est présentée. En cela, les œuvres variables transforment la pratique des restaurateurs/ conservateurs, certains la voyant même se développer vers l'ethnographie afin qu'ils puissent conserver les deux aspects de l'œuvre, tangible et intangible ${ }^{51}$. Par ailleurs, cette documentation se crée dans la durée et s'étoffe périodiquement, presque à chaque fois que l'œuvre est présentée. Les savoirs de l'œuvre se transmettent dans sa pratique, ce qui conduit à prendre des décisions au cas par cas ${ }^{52}$. Nous avons également vu que certains médiateurs institutionnels (Christian Bernard, Anne-Marie Ninacs) développent une approche proactive de la conservation et transforment leur pratique à cause des relations qu'ils tissent avec les œuvres et les artistes.
50

Voir HUMMELEN, ljsbrand et Tatja SCHOLTE. "Sharing Knowledge for the Conservation of Contemporary Art: Changing Role in a Museum Without Walls?». In. HUMMELEN, Ijsbrand et Dionne SILLE (dir.). Modern Art Who Cares? Amsterdam: The Foundation for the Conservation of Modern Art and the Netherlands Institute for Cultural Heritage, 1999, p. 208-212.

51

VAN SAAZE, Vivian. "From Intention to Interaction: Reframing The Artist's Interview in Conservation Research". In. Institut national du patrimoine. Art d'aujourd'hui, patrimoine de demain. Conservation et restauration des œuvres contemporaines. Paris: Institut national du patrimoine, 2009, p. 20-28.

52

Renée VAN DE VALL ("Towards a Theory and Ethics for the Conservation of Contemporary Art». In. Institut national du patrimoine. Art d'aujourd'hui, patrimoine de demain. Conservation et restauration des œuvres contemporaines. Paris: Institut national du patrimoine, 2009, p. 51-56) associe fort à propos cette manière de travailler et de penser à la casuistique. 
53

Carol Mancusi-Ungaro, directeur associé au laboratoire de restauration du Whitney Museum à New York, a fait figure de pionnière avec ses longues entrevues filmées des artistes en présence de leur œuvre. Elle s'est exprimée à plusieurs reprises sur sa pratique. (Voir. par exemple, "Original Intent: The Artist's Voice". In. HUMMELEN et SILLE, Modern Art who cares?, op. cit., p. 392-393.)

54

A titre exemplaire, faisons référence à la base d'archives commencée en 2002 par l'International Network for the Netherlands Institute for Cultural Heritage (INCCA), qui répertorie toute la documentation détenue par ses membres. (Voir http://www.incca.org/> (consulté le 5 août 2010.)
Notre étude mène aussi à cerner le rôle central de l'artiste dans l'espace de la diffusion où il a transporté la production. Même si la documentation est constituée, on continue de s'adresser à lui, que ce soit au MNBAQ ou au MBAC. L'acquisition entraîne une collaboration cyclique à long terme. L'authenticité de l'œuvre ne reposant plus uniquement sur sa technique et sa matérialité, on s'appuie sur l'artiste, la personne de référence quant à l'intégrité conceptuelle, car il figure à l'origine de l'œuvre, il est la source primaire. Dans les pratiques de conservation/restauration de l'art contemporain, l'intention de l'artiste est devenue une valeur culte. Cela se remarque notamment par le rôle grandissant des questionnaires et des entretiens d'artistes depuis les années 1960. Les questionnaires techniques se sont étoffés en s'intéressant davantage à l'aspect conceptuel de l'œuvre et même à l'ensemble de la pratique de l'artiste ${ }^{53}$. De même, les modes d'enregistrement de la parole ont évolué. De notes manuscrites, on est passé aux enregistrements vocaux puis aux captations vidéo afin que l'artiste transmette tous ses savoirs. Les propos et les gestes des artistes prennent de l'importance au point d'en réunir les références dans des bases de données professionnelles que les musées consultent afin de guider leurs décisions ${ }^{54}$. Il ne s'agit plus de notes techniques sur une œuvre précise, mais bien d'un processus créatif qu'on essaie de cerner. 

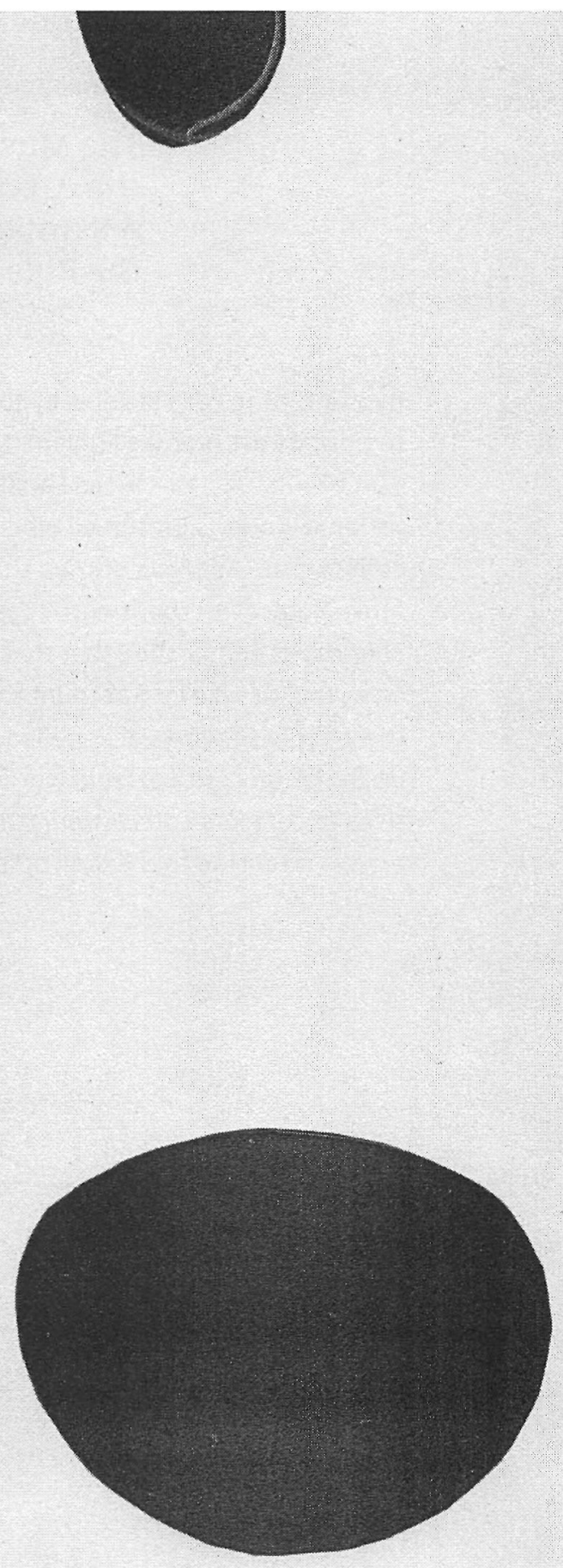


\section{Summary \\ Translated by Philippe Lagrange \\ The Museum Collection and the Artwork's Variability: A Sample of Practices and the Role of the Artist}

By examining case studies by four artists in the field of contemporary art (Karilee Fuglem, Massimo Guerrera, Sarkis and Annie Thibault), this article analyses how the integrity of the variable works is preserved when they belong to institutional collections. Analysis shows that there are no generalized museological methods with regard to the acquisition of variable works and further, that these transform museum culture: by having to take into account the work's conceptual integrity these types of works acknowledge the importance of the artist in the process, variable works modify the respective practices of curators and conservators. This, in turns elevates the artist's intention to the rank of sacred value. 